
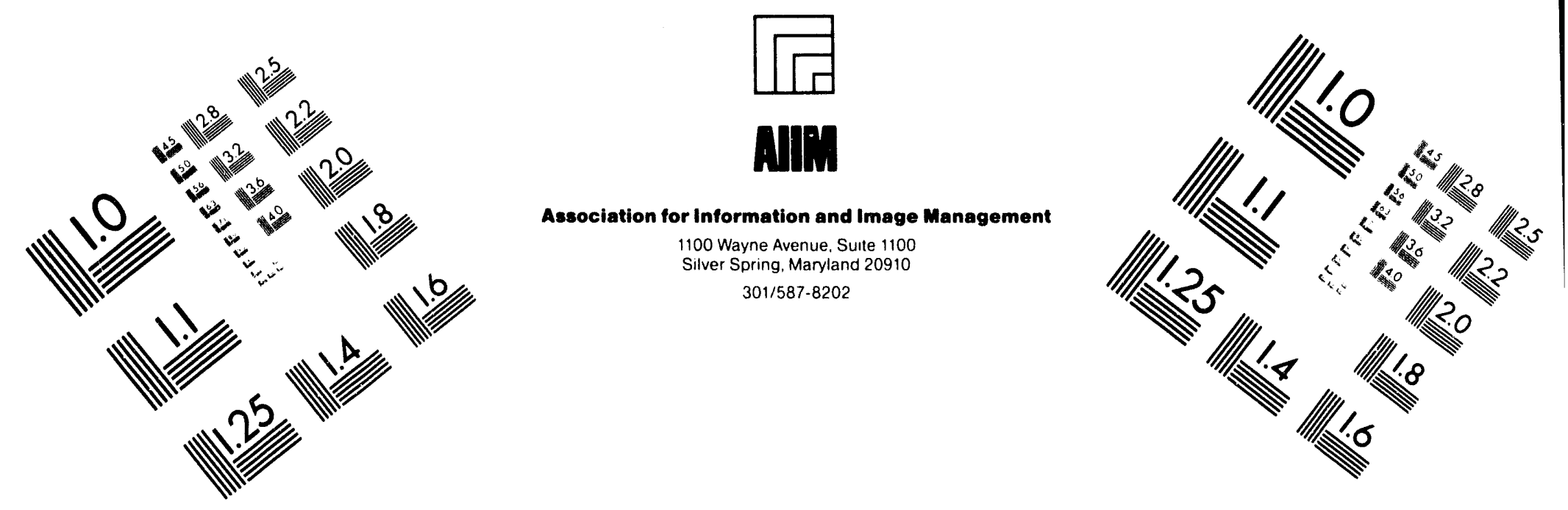

\title{
Centimeter
}

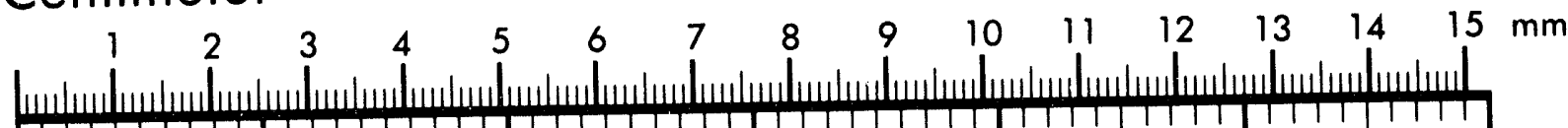

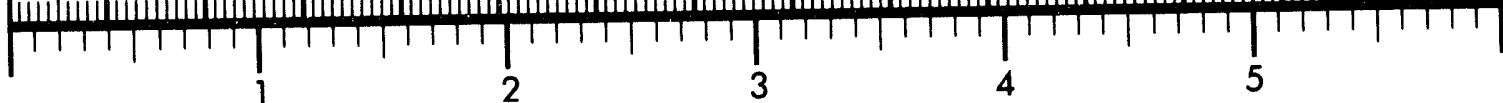
Inches
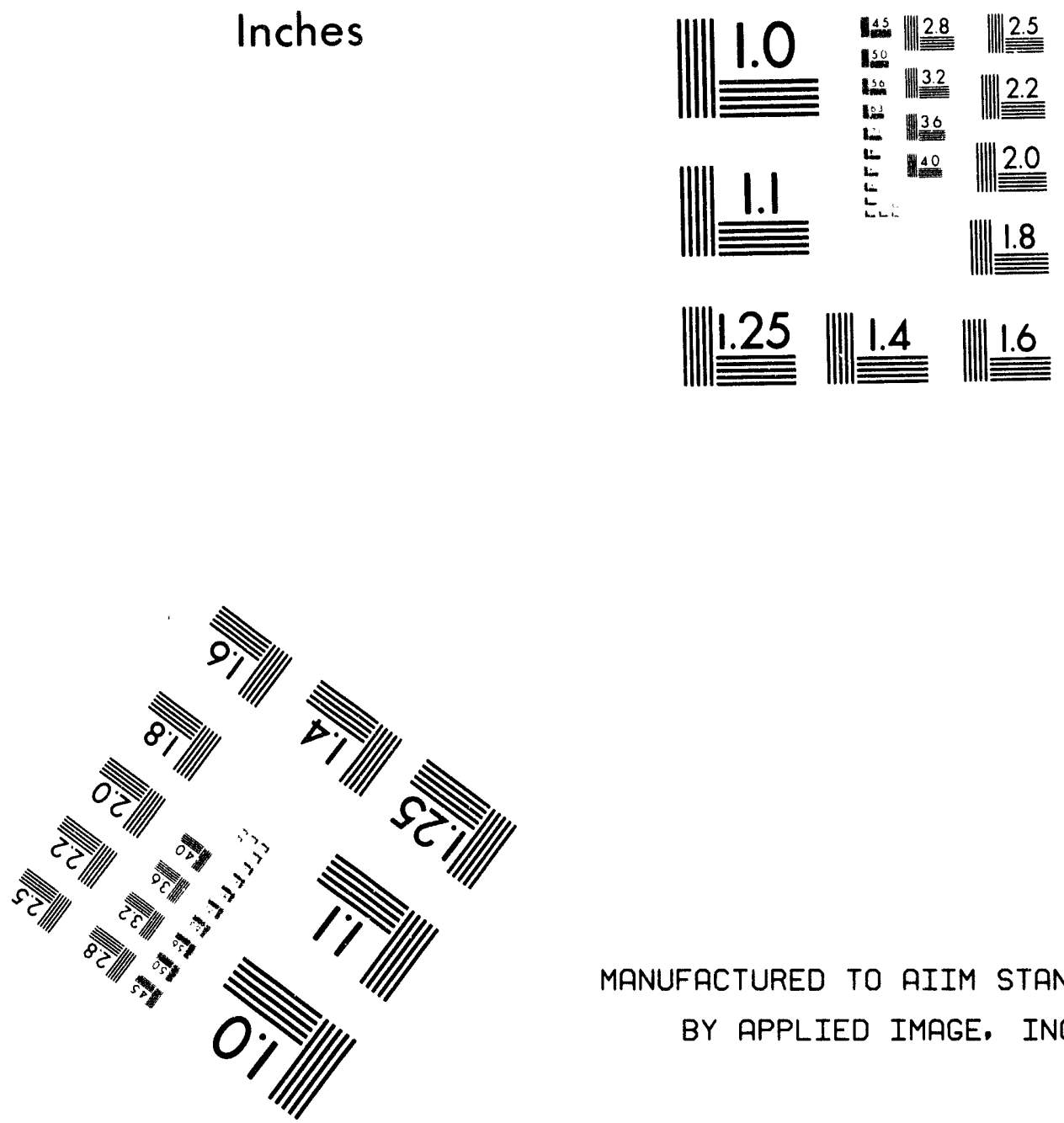

MANUFACTURED TO AIIM STANDARDS BY APPLIED IMAGE, INC.

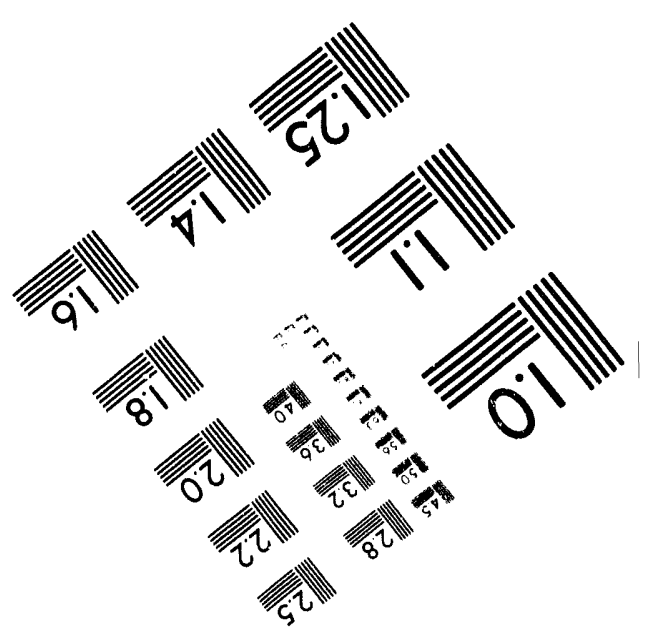



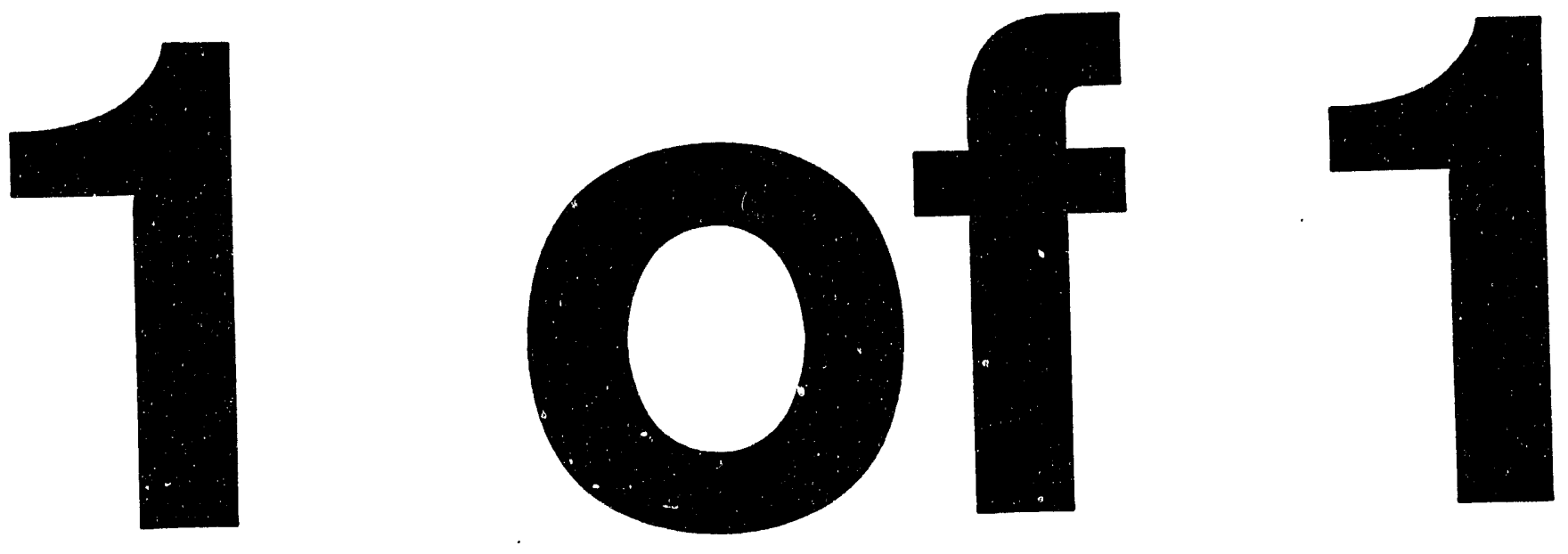


\section{Carrier Doping and Interlayer Coupling in HTSC Single Crystals}

K. Kishio ${ }^{a}$, J. Shimoyama ${ }^{a}$, T. Kimura ${ }^{a}$, Y. Kotaka ${ }^{a}$, K. Kitazawa ${ }^{a}$, K. Yamafujib, Q. $\mathrm{Li}^{c}$ and M. Suenagac

a Department of Applied Chemistry, University of Tokyo, Hongo, Tokyo 113, Japan

'Department of Electronics, Kyushu University Hakozaki, Fukuoka 812, Japan

${ }^{c}$ Division of Materials Sciences, Brookhaven National Laboratory, Upton, New York 11973, U.S.A.

Experimental results of the effect of carrier doping on the irreversibility lines in $(\mathrm{La}, \mathrm{Sr})_{2} \mathrm{CuO}_{4-\delta}$ and $\mathrm{Bi}_{2} \mathrm{Sr}_{2} \mathrm{CaCu}_{2} \mathrm{O}_{8+\delta}$ single crystals are summarized. As a function of $\mathrm{Sr}$ or oxygen contents, systematic and dramatic widening of the irreversible regions in the $B-T$ phase diagram was observed in both systems. The present study suggests the critical importance of carrier concentration which directly affects the interlayer coupling strength and dimensionality of the flux line lattice in all the layered HTSC compounds as a universal feature.

In the HTSC cuprates, electromagnetic anisotropy and the strength of the interlayer coupling along the layer stacking direction (//c-axis) are known to affect the reversible and irreversible magnetic behaviors in the mixed state. While the electrical anisotropy is determined essentially by the structural feature; the interlayer distance of the $\mathrm{CuO}_{2}$ planes and the chemical nature of the "blocking layer", it is also expected to be affected by carrier doping. However, how the doping state of a given compound affects its magnetic properiies has not been investigated in detail.

In this paper, irreversibility lines of highquality single crystals of $\left(\mathrm{La}_{1-x} \mathrm{Sr}_{x}\right)_{2} \mathrm{CuO}_{4-\delta}$ (LSCO) as well as $\mathrm{Bi}_{2} \mathrm{Sr}_{2} \mathrm{CaCu}_{2} \mathrm{O}_{8+\delta}(\mathrm{Bi2212})$ over wide ranges of carrier doping states, are compared and the universal features, common in these layered HTSC cuprates, are addressed. LSCO crystals $(x=0.05,0.0625,0.085$ and 0.10 ) have been prepared by the TSFZ technique and their electrical anisotropy as well as reversible magnetic properties have been extensively investigated as described elsewhere $[1,2]$. Bi2212 crystals were prepared similarly by the FZ technique and their oxygen contents were carefully controlled by low temperature annealing in sealed quartz tubes [3].

Figure 1 summarizes the observed irreversibility points of LSCO determined from $z f c-f c$ curves by SQUID (open symbols) and $M-H$ hysteresis curves by VSM magnetometry (closed symbols) with a sweep rate of $20 \mathrm{mT} / \mathrm{s}$.
It should be noted that the temperature scale has been normalized with respect to $T_{c}$ of each crystal, $26.2,32.4,35.0$ and $29.8 \mathrm{~K}$ with an increasing order in $x$. The difference in time scale of the measurements between the two methods can be seen to be insignificant. It is immediately found that the irreversibility lines are located at higher fields in the crystals with larger $\mathrm{Sr}$ contents. Since the temperature slope of the mean field $B_{c 2}$ values, $d B_{c 2} / d T$, has been found to be approximately same, $-1 \sim 1.5 \mathrm{~T} / \mathrm{K}$, in these samples [2], Fig. 1 clearly sugrests that the widening of the irreversible region in the $B-T$ plane is taking place as a function of $x$.

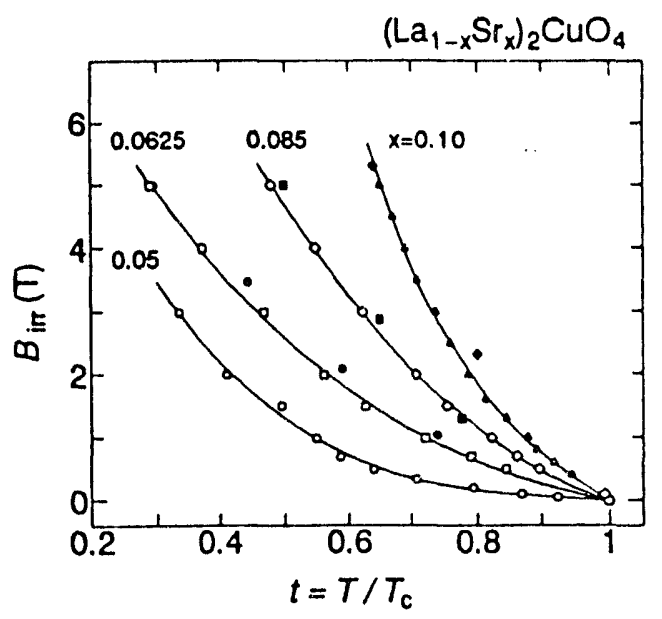

Figure 1. Irreversibility field $(\mathrm{H} / / \mathrm{c})$ as a function of reduced temperature in LSCO single crystals with various $\mathrm{Sr}$ contents, $x$.

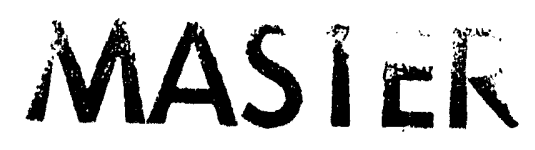




\section{DISCLAIMER}

This report was prepared as an account of work sponsored by an agency of the United States Government. Neither the I'nited States Government nor any agency thereof, nor any of their employees, makes any warranty, express or implied, or assumes any legal liability or responsibility for the accuracy, completeness, or usefulness of any information, apparatus, product, or process disclosed, or represents that its use would not infringe privately owned rights. Reference herein to any specific commercial product, process, or service by trade name, trademark, manufacturer, or otherwise does not necessarily constitute or imply its endorsement, recommendation, or favoring by the United States Government or any agency thereof. The views and opinions of authors expressed herein do not necessarily state or reflect those of the United States Government or any agency thereof. 


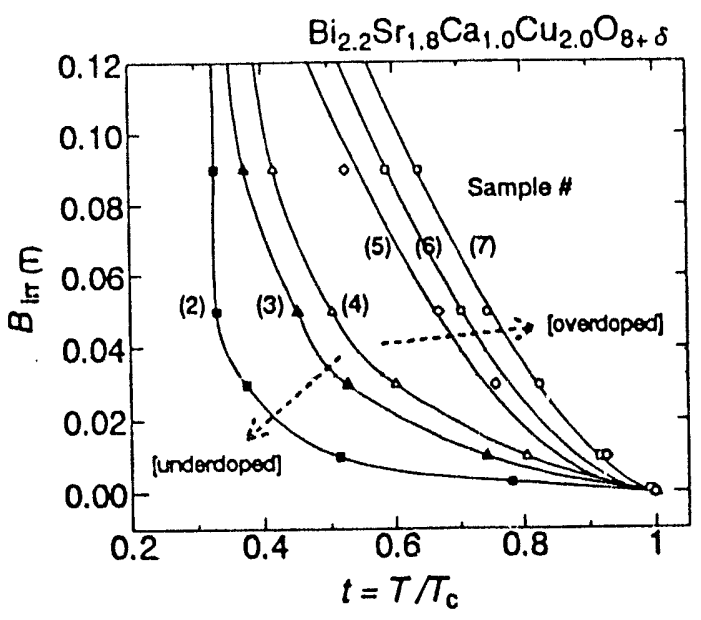

Figure 2. Irreversibility field $(\mathrm{H} / / \mathrm{c})$ as a function of reduced temperature in Bi2212 single crystals with various oxygen content.

Figure 2 summarizes the irreversibility lines of Bi2212 crystals having various oxygen contents, $\delta[4]$. $\delta$ increases for larger sample number; \#2 being most underdoped and \#7 is most overdoped, where the difference of oxygen content, $\Delta \delta$, is estimated to be $\sim 0.05$ between the two. Along with the increase in $\delta, T_{c}\left(\chi_{\text {mid }}\right)$ varies from $76.0 \mathrm{~K}(\# 2)$, through $86.4 \mathrm{~K}(\# 4)$ and to $76.7 \mathrm{~K}(\# 7)$. While $B_{\text {irr }}$ is shown here again with a reduced temperature scale as was in Fig. 1, it must be noted that the distinctively higher $B_{\text {irr }}$ lines for \#5 7 is not a result of this procedure. Even when plotted against the normal temperature scale, $B_{\text {irr }}$ has been found to have the steeper slope for the more overdoped crystal [4].

The close resemblance of Figs, 1 and 2 can be understood in terms of the composition dependence of the carrier doping states in these two compounds. In both systems, it has been well-demonstrated that normal state resistivity both along the c-axis, $\rho_{c}$, and the ab-plane, $\rho_{a b}$, as well as their anisotropy ratio, $\rho_{c} / \rho_{a b}$, are monotonically decreased with the $\mathrm{Sr}$ content in LSCO [1] and with the oxygen content in $\mathrm{Bi} 2212$ [3]. Based on this consideration, we have plotted $B_{\text {irr }}$ at $t \equiv T / T_{c}=0.7 \sim 0.9$ of both systems as a function of $\gamma^{2} \equiv \rho_{c} / \rho_{a b}$ evaluated at $T_{c}$ onset temperatures. The results are shown in Fig. 3, including the reported $B_{\text {irr }}$ of YBCO single crystals in the literature [5] with their estimated $\gamma^{2}$.

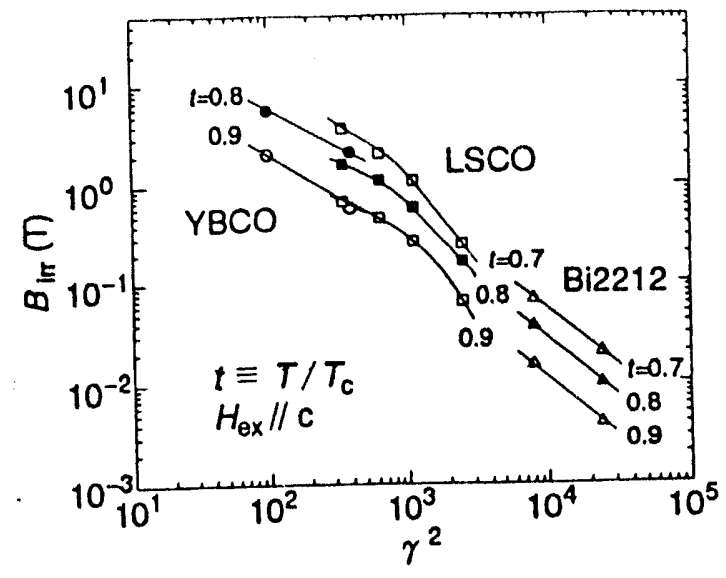

Figure 3. Irreversibility field $(\mathrm{H} / \mathrm{c})$ at various reduced temperatures of $\mathrm{YBCO}$, LSCO and Bi2212 plotted as a function of their electrical anisotropy, $\gamma^{2}\left(\equiv \rho_{c} / \rho_{a b}\right)$.

It is particularly interesting that $B_{i r r}$ changes quite rapidly with $\gamma^{2}$ and all the curves correlate rather well in the three systems extending over an extremely wide range of $\gamma^{2}$ values.

It has been believed that the weak pinning behavior of the Bi2212 compound originate from the short correlation length, $\ell_{44}$, of the flux line lattice along the layer stacking direction because of the weak interlayer coupling (Josephson coupling) of the $\mathrm{CuO}_{2}$ planes across the $\mathrm{Bi}_{2} \mathrm{O}_{2}$ layers. $A$ recent microwave penetration measurement [6] has also shown that the interlayer coupling in the LSCO system is also Josephson-like. Figure 3 seems to suggest that such a picture should be correct in all of the layered HTSC cuprate systems and strengthening of the interlayer coupling by carrier doping is extremely effective, in order to improve the flux pinning properties of these materials.

\section{REFERENCES}

1. T. Kimura et al, Physica C192 (1992) 247.

2. Q. Li et al, Phys. Rev. B47 (1993) 11384.

3. Y. Kotaka et al, Physica C, Present Volume.

4. K. Kishio et al, Proc. 7th IWCC (1994), Jan. 24-27, Alpbach, Austria, in press.

5. M. A. Angadi et al, Physica C185-189 (1991) 2159

6. T. Shibauchi et al, Phys. Rev. Lett. 72 (1994) 2263.

\section{ACKNOWLEDGMENT}

This research was performed under the auspices of the U.S. Department of Energy, Division of Materials Sciences, Office of Basic Energy Sciences under Contract No. DEAC02-76CH0O016. 

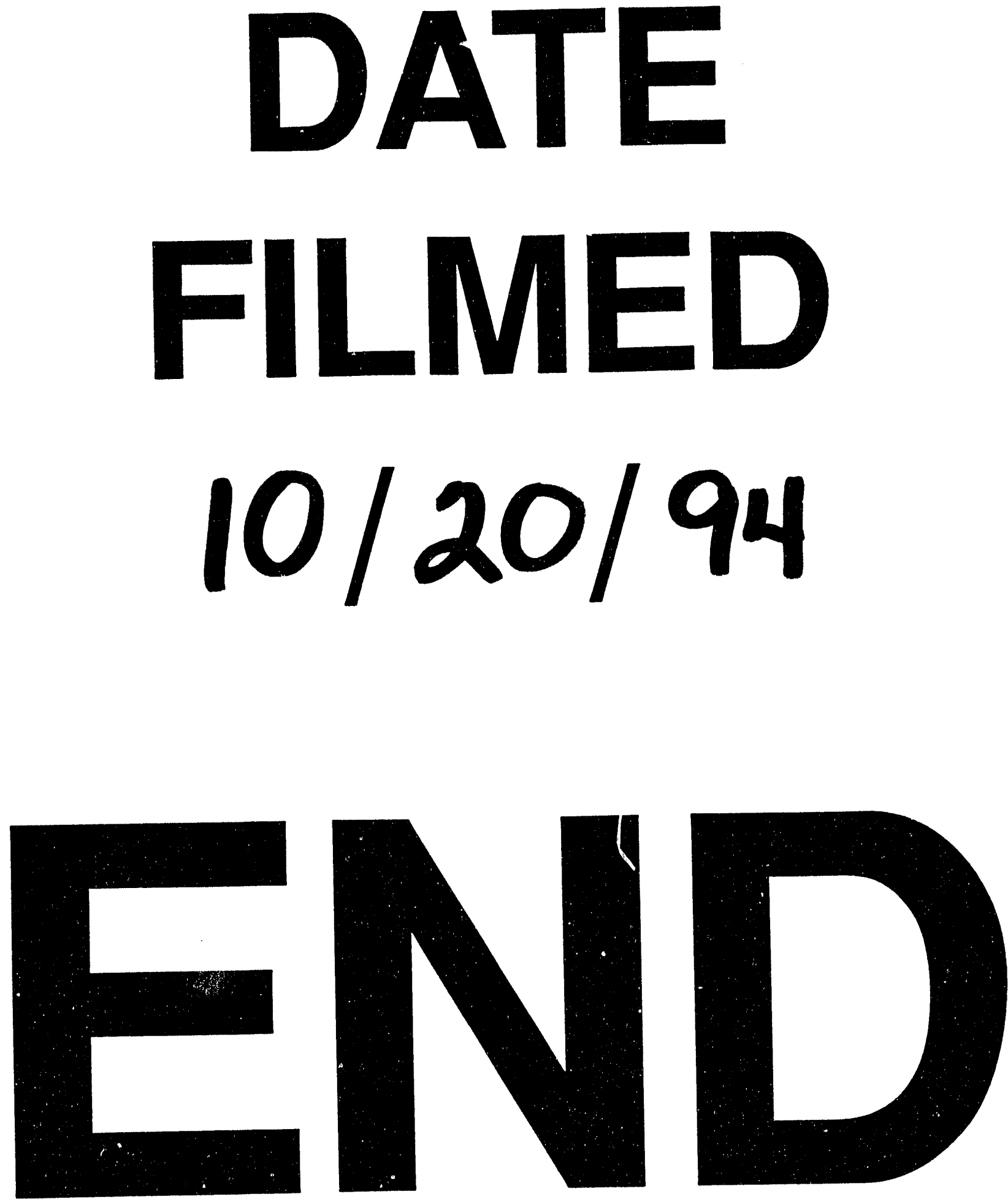
